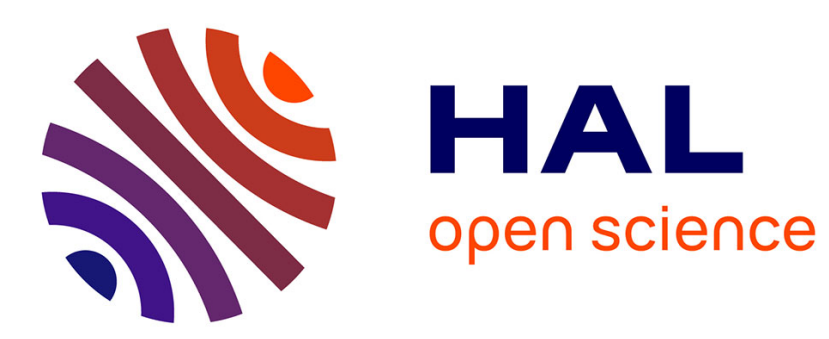

\title{
A Parallel Adaptive Projection Method for Incompressible Two Phase Flows
}

Davide Zuzio, Jean-Luc Estivalezes

\section{To cite this version:}

Davide Zuzio, Jean-Luc Estivalezes. A Parallel Adaptive Projection Method for Incompressible Two Phase Flows. ICCFD6 (6th International Conference on Computational Fluid Dynamics), Jul 2010, SAINT PETERSBOURG, Russia. hal-02178687

\section{HAL Id: hal-02178687 \\ https://hal.science/hal-02178687}

Submitted on 10 Jul 2019

HAL is a multi-disciplinary open access archive for the deposit and dissemination of scientific research documents, whether they are published or not. The documents may come from teaching and research institutions in France or abroad, or from public or private research centers.
L'archive ouverte pluridisciplinaire HAL, est destinée au dépôt et à la diffusion de documents scientifiques de niveau recherche, publiés ou non, émanant des établissements d'enseignement et de recherche français ou étrangers, des laboratoires publics ou privés. 


\title{
A Parallel Adaptive Projection Method for Incompressible Two Phase Flows
}

\author{
$\underline{\text { Davide Zuzio }^{1}}$, Jean-Luc Estivalezes ${ }^{1}$
}

\begin{abstract}
Direct numerical simulation of high-density ratio multiphase flows requires a lot of computational resources. We have developed a parallel algorithm for solving the incompressible Navier-Stokes equation in two-phase flows on an adaptive hierarchy of mesh. The interface between the two fluids is treated by a coupled Level-Set/Ghost-Fluid method; the parallel AMR is handled by the PARAMESH package, which assures good scaling. A robust solver for the variable density pressure equation has been developed, including an AMR levels-based multigrid preconditioner. The code has been applied to the study of the oscillation of a two dimensional liquid sheet sheared by an air flow, and global oscillation frequencies have been computed for a simplified test case. A high resolution computation has been performed to show two dimensional ligament formation and break-up.
\end{abstract}

\section{Introduction}

In engineering the study of liquid-gas interactions is important in combustion problems: the formation of droplets clouds or sprays that burn in combustion chambers are originated by interfacial instabilities, such as the Kelvin-Helmholtz instability. In the case of airblast type injectors the shearing effect of the airflow provokes a longitudinal instability, which in turn originates a transversal one, a process that leads to the formation of ligaments and droplets. The numerical simulation can be a powerful tool for predicting the behaviour of these physical phenomena, especially in scales of time or space where experimental visualization is difficult or impossible. In a two fluid configuration the adaptive mesh is naturally advisable, as if the precision of the interface discretization depends on the size of the cells, it is often necessary to extend the computational domain well beyond the interfacial region of the flow, thus

Davide Zuzio, Department of Models For Aerodynamics and Energy, ONERA, Toulouse, France, e-mail: davide.zuzio@onecert.fr·Jean-Luc Estivalezes, Department of Models For Aerodynamics and Energy, ONERA, Toulouse, France, e-mail: jean-luc.estivalezes@onera.fr 
involving a lot of computational effort spent on less interesting zones. The mesh is spatially adaptive: the domain is divided in blocks all having the same number of cells, which can be bisected in each directions when needed. This approach intuitively gives a lighter dedicated tree memory storage, an easier cache managing and parallelization than other kind of adaptive mesh. The adaptive mesh represents here an improvement over the algorithm of Couderc and Estivalezes (2003), and retains its Cartesian structured mesh discretization. The PARAMESH libraries developed by MacNeice et al (2000) are here employed, designed to provide to an application developer an easy route to extend an existing code which uses a logically Cartesian structured mesh into a code with parallelized AMR.

\section{Physical model}

The incompressible Navier-Stokes equations are solved in each of the two non miscible fluids.

$$
\begin{aligned}
\nabla \cdot \boldsymbol{u} & =0 \\
\frac{\partial \boldsymbol{u}}{\partial t}+(\boldsymbol{u} \cdot \nabla) \boldsymbol{u} & =\frac{1}{\rho}(\nabla \cdot \mathbf{T}+f)
\end{aligned}
$$

The computational domain is divided in two regions separated by a sharp interface. Across the interface, the jump conditions are imposed by capillarity and viscosity; the velocity respects a no-slip condition.

$$
\begin{aligned}
{[p]-\mathbf{n} \cdot\left[\mu\left(\nabla \boldsymbol{u}+(\nabla \boldsymbol{u})^{t}\right)\right] \cdot \mathbf{n} } & =\sigma \kappa \\
\mathbf{t} \cdot\left[\mu\left(\nabla \boldsymbol{u}+(\nabla \boldsymbol{u})^{t}\right)\right] \cdot \mathbf{t} & =0 \\
{[\boldsymbol{u}] } & =0
\end{aligned}
$$

For the interface tracking a Level Set approach is used. The interface is implicitly given by the zero level of a function $\phi(\mathbf{x}, t)$ where $\phi$ is defined as the signed distance to the interface. The evolution of the Level Set follows a linear advection equation so that it is passively advected by the velocity field.

$$
\frac{\partial \phi}{\partial t}+\boldsymbol{u} \cdot \nabla \phi=0
$$

From the value of the function the normal vector and the curvature can be easily derived. 


\section{Single grid solver}

\section{Advection of the Level Set}

The Level Set equation is solved by a finite volumes type spatial discretization, that assures the conservation of the integral quantity. The approximation of the numerical fluxes is based on an "upwinding" method which, conversely to the centered schemas, gives a convergent discretization under opportune CFL condition: the approximation of the fluxes is given by a Weno5 type discretization by Liu et al (1994). The temporal discretization of the time derivative is done by a third order RungeKutta scheme as described by Shu and Osher (1988).

\section{Resolution of momentum equation}

The momentum equation is solved by a two step projection method described by Chorin (1968). This method de-couples velocity and pression by using first an explicit discretization for the effects of convection in a predictor step, and then enforcing the compliance with a velocity divergence constraint in a subsequent projection step. The discretization of the viscous terms is totally explicit second order centered scheme. As for the Level Set function, the Weno5 scheme is used for the spatial discretization of the advective term. The temporal integration of the momentum equation is realized by a second order Adams-Bashford scheme. A standard five points, second order centered discretization is used for the Laplacian operator in equation. In order to ensure the temporal stability of the explicit projection step, an adaptative time step $\Delta t^{n}$ in respect of the convective, viscous, gravitational and capillary effects is defined.

\section{Jump conditions}

The approach used for the application of jump conditions is the Ghost Fluid method, developed by Kang et al (1999). It gives a discretization for discontinuous numerical derivatives across a sharp interface. In order to evaluate the first and second derivatives, the jump values in $C^{0}$ and $C^{1}$ are introduced into the definitions of the derivatives themselves. Those values allow the computation of a projected value of the quantity to the other side of the discontinuity, finally completing the discretization stencil. 


\section{AMR}

In the present work, the PARAMESH package (MacNeice et al (2000)) has been chosen to be used with the code. It is an open-source software package which manages the creation of a block-type AMR: builds and maintains the tree-structure which tracks the spatial relationships between blocks, distributes the blocks amongst the available processors and handles all inter-block and inter-processor communication. It can distribute the blocks so that it can maximize block locality and minimize inter-processor communications. Each block is an uniform mesh with user defined dimensions. The refinement ratio is fixed to $r=2$. PARAMESH parallelization is done via MPI; the repartition of the workload is realized by equally distributing the blocks among the processors, as each block requires the same computational effort. In the Level Set formulation, the chosen refinement criterion is the proximity of the interface.

\section{Poisson solver}

The BiCG-Stab solver (VanDerVorst (1992)) has been chosen because of its ability to deal with unsymmetrical systems, its stability, and the regularity of convergence; moreover, it can take advantage from the multigrid acceleration. The solver preconditioner was built using the PARAMESH Multigrid routines; the chosen algorithm is known in literature as FAC (Fast Adaptive Composite grid solver, McCormick and Thomas (1986)). In this algorithm the relaxation sweeps are performed across the entire computational domain, and one defines the succession of multigrid levels by restricting the highest resolution sub-grids to the newt lower resolution, actually working on composite grids (grids composed by a union of blocks usually not at the same refinement level). Some attention has to be paid to the coarse-fine interface: here the continuity of the solution first derivative should be imposed (elliptic matching condition); otherwise the solver convergence may stagnate, and the solution may not benefit from the refined grids.

\section{Numerical results: primary atomization of a liquid sheet}

In this test results from the simulation of the primary atomization of a liquid sheet sandwiched between two parallel air streams are given. In this configuration, the sheet-shape is unstable. The hydrodynamic instabilities such as the KelvinHelmholtz are the source of the atomization mechanisms formation and determine the primary break-up characteristics. The first oscillation in the stream wise direction causes the liquid sheet to wave in and out between the two airflows. The secondary oscillation is a three-dimensional phenomenon, as it develops in the span wise direction. The simulation parameters are: $L_{x}=L_{y}=3 \mathrm{~mm}, \rho_{l}=1000 \mathrm{~kg} \cdot \mathrm{m}^{-3}$, 
$\rho_{g}=12.26 \mathrm{~kg} \cdot \mathrm{m}^{-3}, \mu_{l}=1.132 \times 10^{-2} \mathrm{~kg} \cdot \mathrm{m}^{-1} \cdot \mathrm{s}^{-1}, \mu_{g}=1.78 \times 10^{-3} \mathrm{~kg} \cdot \mathrm{m}^{-1} \cdot \mathrm{s}^{-1}$, $\sigma=7.28 \times 10^{-2} \mathrm{~N} . \mathrm{m}^{-1}, a_{\text {sheet }}=150 \times 10^{-6} \mathrm{~m}, \delta=3 \times 10^{-4} \mathrm{~m}, e=1.5 \times 10^{-4}$ $\mathrm{m}$, which correspond to water and pressurized air (10 bar). Here $e$ represents the gas stream thickness without boundary layer, and $\delta$ the thickness of the boundary layer. For the reference computation the injection velocities at infinity are $U_{g}=30$ $\mathrm{m} . \mathrm{s}^{-1}$ and $U_{l}=2 \mathrm{~m} . \mathrm{s}^{-1}$. The viscosities do not represent the real air and water ones, as they have been raised in order to lower the Reynolds number and stabilize the computations. Inflow condition are imposed on the left face, outflow elsewhere. The liquid velocity follows a laminar plane Poiseuille profile, the gas velocity a turbulent Polhausen profile ised by Couderc (2007).

$$
\begin{aligned}
u(y) & =\frac{3 U_{l}}{2 a^{2}}\left(a^{2}-y^{2}\right) \\
\frac{u(y)}{U_{g}} & =2 \frac{y}{\delta}-2\left(\frac{y}{\delta}\right)^{2}+\left(\frac{y}{\delta}\right)^{4}
\end{aligned}
$$

The main interest of the two dimensional simulations is the possibility to isolate the global oscillation phenomenon, as well as the influence of the inflow configuration (figure1). Result show a slight overestimation of the frequencies if compared to experiments, as the ones by Fernandez (2009) and Lozano et al (1998). The high resolution simulation, shown in figure 3 , shows two dimensional phenomena of ligaments formation and break up and the subsequent formation of droplets. Here the viscosities are the real ones for kerosene and water $\left(\mu_{l}=2.3 \times 10^{-3} \mathrm{~kg} . \mathrm{m}^{-1} . \mathrm{s}^{-1}\right.$, $\left.\mu_{g}=1.56 \times 10^{-5} \mathrm{~kg} \cdot \mathrm{m}^{-1} \cdot \mathrm{s}^{-1}\right)$, and the computational domain is more realistically $L_{x}=L_{y}=16 \mathrm{~mm}$ thanks to the AMR. Preliminary investigation of the oscillation frequency suggest better approximation of the realistic values. After the ligament formation the simulation shows a break-up of these, with successive formation of a droplet cloud. The physics, however, is still not close to reality because an important role in the droplet generation is played by the transversal instabilities. The mean droplet size (around 20 microns) is quite small compared to experiments (around 80100 microns from (Fernandez (2009))). The total number of points used is roughly $n p \approx 2 \times 10^{6}$ versus an hypothetical $n p \approx 3 \times 10^{8}$ in case of uniform mesh, a reduction which allows the computation to run on 128 processors instead of about 8192 for best parallel efficiency.

\section{Conclusions}

A second order parallel adaptive projection method has been developed to solve incompressible two phase flows. The ability to perform high density ratio computations has been demonstrated, in particular the ability of the elliptic solver to converge for all the test cases. Results suggest that the AMR simulations can almost achieve the fine grid precision in all the proposed tests, as well as maintaining good convergence rate. Simulations of the two dimensional primary atomization have shown the unstable behaviour of the planar sheet configuration when sheared 
by an air flow with varying injection velocities. An high resolution simulation done with a heavier use of adaptative mesh shows the two dimensional break up of the sheet as well as the formation of a cloud of droplets. A numerical method improvement would allow for lower resolution computation, as well as three dimensional ones.

\section{Acknowledgements}

This research project has been supported by a Marie Curie Early Stage Research Training Fellowship of the European Community's Sixth Framework Programme under contract number MEST-CT-2005-020426.

The PARAMESH software used in this work was developed at the NASA Goddard Space Flight Center and Drexel University under NASA's HPCC and ESTO/CT projects and under grant NNG04GP79G from the NASA/AISR project.

\section{References}

Chorin A (1968) Numerical solution of the navier-stokes equations. Math Comput (22):745-762

Couderc F (2007) Développement d'un code de calcul pour la simulation d'écoulements de fluides non miscibles application à la désintégration assistée d'un jet liquide par un courant gazeux. PhD thesis, ENSAE

Couderc F, Estivalezes JL (2003) Sharp interface capturing method based on levelset method for incompressible flow with surface tension. In: ASME Annual Summer Meeting FEDSM2003-45151, pp 385-393

Fernandez VG (2009) Experimental study of a liquid sheet disintegration in a high pressure environment. PhD thesis, ISAE

Kang M, Fedkiw RP, dong Liu X (1999) A boundary condition capturing method for multiphase incompressible flow. J Sci Comput 15:323-360

Liu X, Osher S, Chan T (1994) Weighted essentially non-oscillatory schemes. Journal of Computational Physics 115:200-212

Lozano A, Barreras F, Hauke G, Dopazo C (1998) Longitudinal instabilities in a liquid sheet. Journal of Fluid Mechanics 39:127-139

MacNeice P, Olson K, Mobarry C, deFainchtein R, Packer C (2000) Paramesh : A parallel adaptive mesh refinement community toolkit. Computer Physics Communications 126:330-354

McCormick S, Thomas J (1986) The fast adaptive composite grid (fac) method for elliptic equations. Applied Mathematics and Computation (46):439-456

Shu C, Osher S (1988) Efficient implementation of essentially non-oscillatory shock capturing schemes. Journal of Computational Physics (77):439-471 
VanDerVorst HA (1992) Bi-cgstab: a fast and smoothly converging variant of bi$\mathrm{cg}$ for the solution of nonsymmetric linear systems. SIAM Journal of Scientific Computing 13:631-644 



\section{List of Figures}

1 Configuration of the sheet and computed signal at different streamwise positions. . . . . . . . . . . . . . . . 8

2 Quantitative results from the sheet simulations: computed oscillation frequencies for different liquid and air speeds. ........ 9

3 High resolution qualitative results: time is increasing from light to intense colour. . . . . . . . . . . . . . . . . . . . . . . . . 10 
Fig. 1: Configuration of the sheet and computed signal at different streamwise positions.
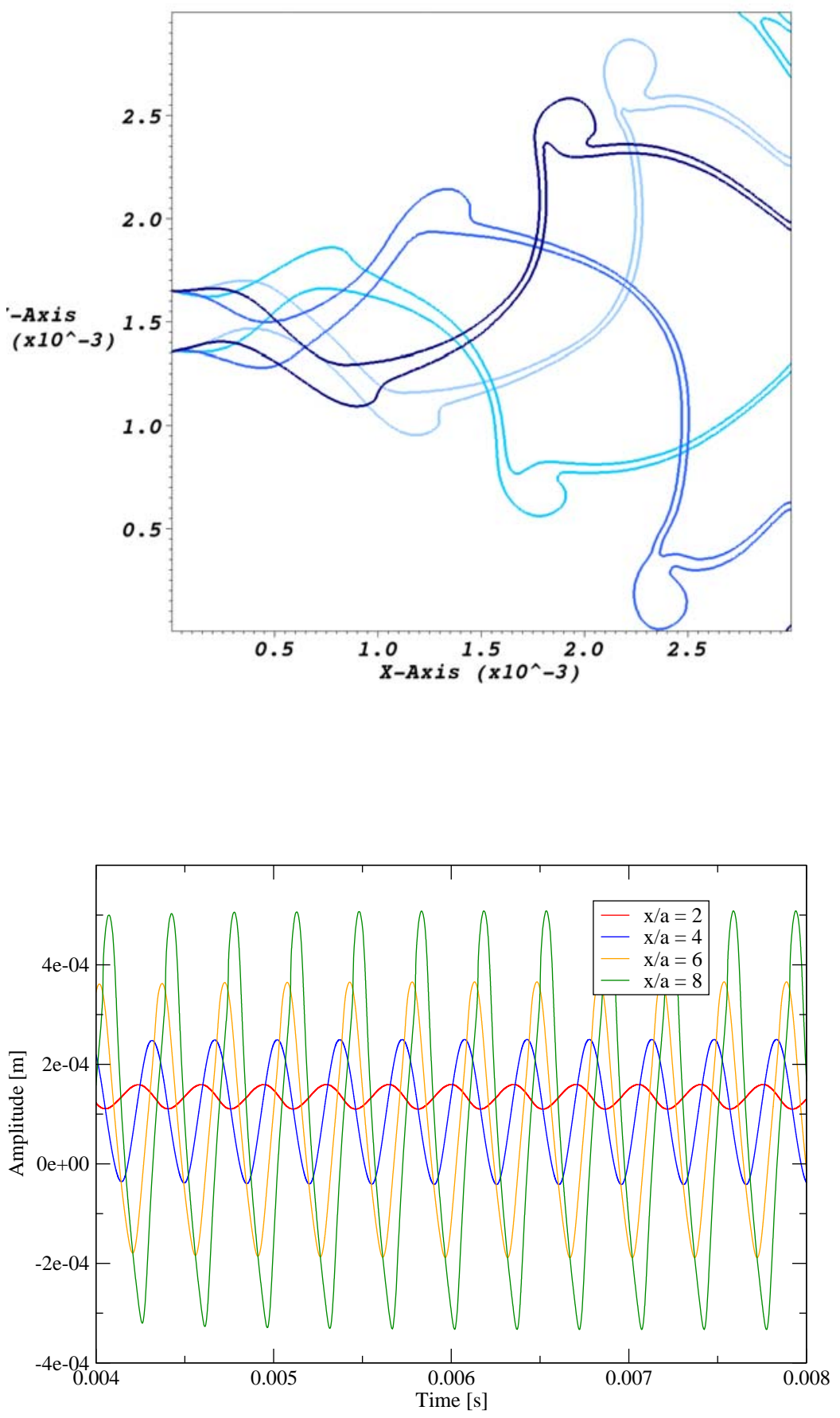


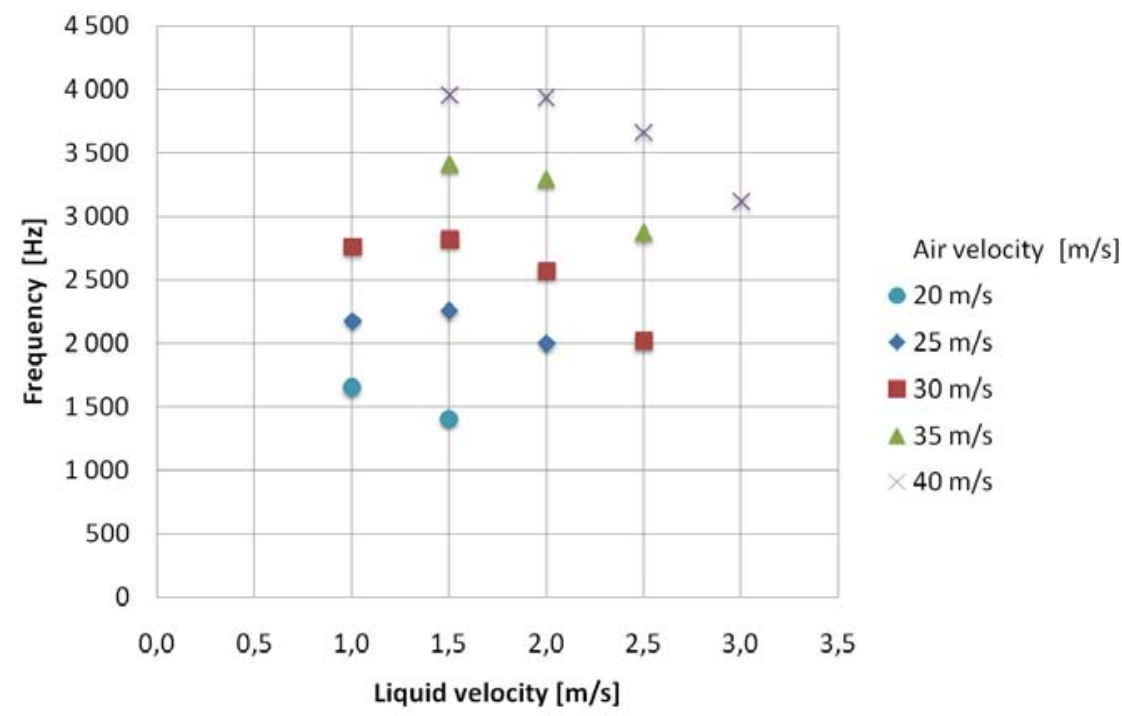

Fig. 2: Quantitative results from the sheet simulations: computed oscillation frequencies for different liquid and air speeds. 


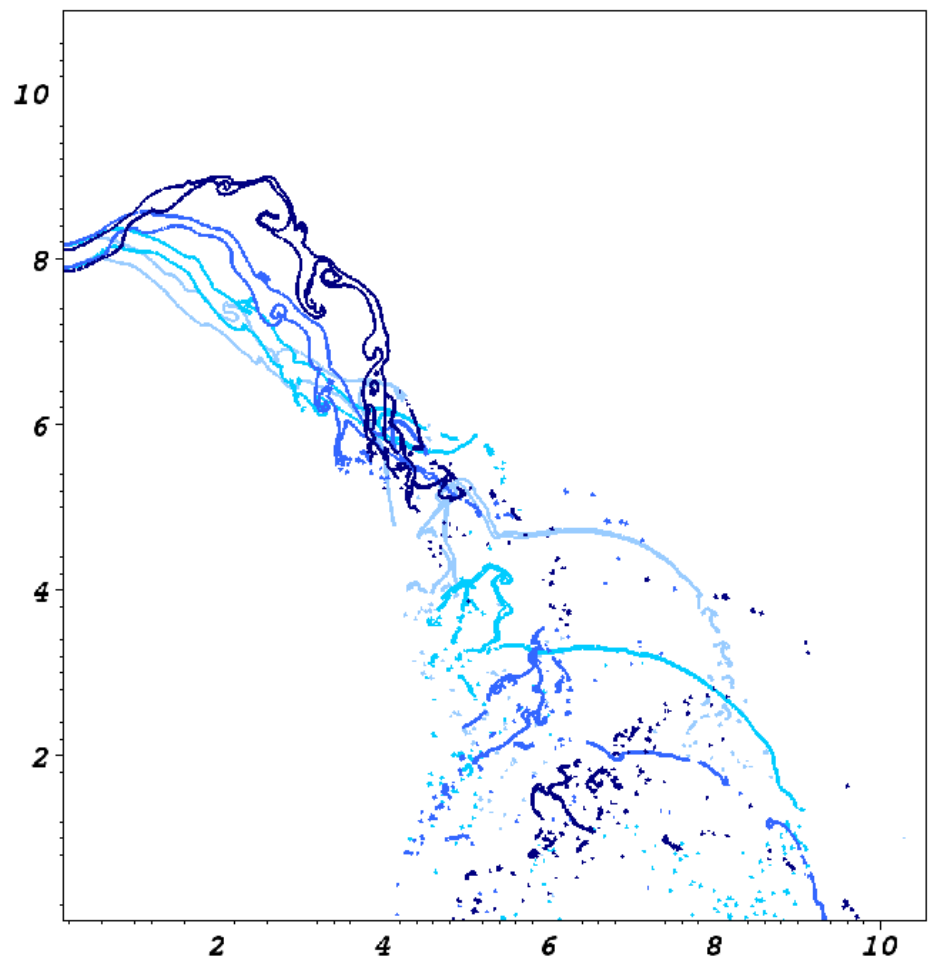

Fig. 3: High resolution qualitative results: time is increasing from light to intense colour. 